\title{
Triggering Receptor Expressed on Myeloid Cells-1 Signaling: Protective and Pathogenic Roles on Streptococcal Toxic-Shock- Like Syndrome Caused by Streptococcus suis
}

OPEN ACCESS

Edited by: Emilio Luis Malchiodi, Universidad de Buenos Aires, Argentina

Reviewed by:

M. Victoria Delpino,

National Scientific and Technical Research Council (CONICET), Argentina Ashok Munjal, Barkatullah University, India

*Correspondence: Anding Zhang

andye8019@mail.hzau.edu.cn

Specialty section: This article was submitted to Microbial Immunology, a section of the journal

Frontiers in Immunology

Received: 04 October 2017 Accepted: 07 March 2018 Published: 21 March 2018

Citation:

Han L, Fu L, Peng $Y$ and Zhang A (2018) Triggering Receptor Expressed on Myeloid Cells-1 Signaling: Protective and Pathogenic Roles on Streptococcal Toxic-ShockLike Syndrome Caused by Streptococcus suis.

Front. Immunol. 9:577. doi: 10.3389/fimmu.2018.00577

\begin{abstract}
Li Han', Lei Fu',2,3,4, Yongbo Peng ${ }^{5}$ and Anding Zhang ${ }^{1,2,3,4 *}$
'State Key Laboratory of Agricultural Microbiology, College of Veterinary Medicine, Huazhong Agricultural University, Wuhan, China, ${ }^{2}$ Key Laboratory of Preventive Veterinary Medicine in Hubei Province, The Cooperative Innovation Center for Sustainable Pig Production, Huazhong Agricultural University, Wuhan, China, ${ }^{3}$ Key Laboratory of Development of Veterinary Diagnostic Products, Ministry of Agriculture, Wuhan, China, ${ }^{4}$ International Research Center for Animal Disease, Ministry of Science and Technology, Wuhan, China, ${ }^{5}$ Institute for Medical Biology, Hubei Provincial Key Laboratory for Protection and Application of Special Plants in Wuling Area of China, College of Life Sciences, South-Central University for Nationalities, Wuhan, China
\end{abstract}

Streptococcus suis infections can cause septic shock, which is referred to as streptococcal toxic-shock-like syndrome (STSLS). The disease is characterized by a severe inflammatory response, multiple organ failure, and high mortality. However, no superantigen that is responsible for toxic shock syndrome was detected in S. suis, indicating that the mechanism underlying STSLS is different and remains to be elucidated. Triggering receptor expressed on myeloid cells-1 (TREM-1), belonging to the Ig superfamily, is an activating receptor expressed on myeloid cells, and has been recognized as a critical immunomodulator in several inflammatory diseases of both infectious and non-infectious etiologies. In this review, we discuss the current understanding of the immunoregulatory functions of TREM-1 on acute infectious diseases and then highlight the crucial roles of TREM-1 on the development of STSLS.

Keywords: triggering receptor expressed on myeloid cells-1, streptococcal toxic-shock-like syndrome, Streptococcus suis, inflammation, cytokine storm

\section{INTRODUCTION}

Streptococcus suis is a major swine pathogenic bacterium, and it is also a severe threat to human health (1-4). Since the first reported case of S. suis-induced meningitis in humans in Denmark in 1968 , more than 1,600 human infection cases have been reported in the world $(5,6)$. In addition, $S$. suis has also been recognized as the leading and second cause of adult meningitis in Vietnam and Thailand, respectively $(1,7,8)$. For a long time, $S$. suis infections in humans have remained sporadic and mainly affect individuals who have closely contacted with pigs or pig-derived products (9-11). However, the two large-scale outbreaks in China $(12,13)$ and human cases without a history of animal contact $(14,15)$ have modified opinion regarding the threat of this pathogen to humans.

Streptococcus suis infections in humans normally produce meningitis, endocarditis, cellulitis, peritonitis, arthritis, pneumonia, and occasionally septic shock, and the pooled case-fatality rate is $12.8 \%(1,16,17)$. Now, special attention is given to the largest outbreak in China in 2005, which 
caused 38 deaths among 204 human infections. Of the 38 deaths, 37 were caused by septic shock, which is designated as "streptococcal toxic-shock-like syndrome (STSLS)” (12). Unfortunately, 63\% of STSLS patients died even after treatment with antibiotics (18), and STSLS is characterized by high serum levels of IFN- $\gamma$, TNF- $\alpha$, IL-8, IL-12, and IL-1 $\beta$, termed "cytokine storm" (19). However, no superantigen that is responsible for toxic shock syndrome was detected in S. suis (12), indicating that the mechanism underlying STSLS is different from that of toxic shock syndrome.

High levels of systemic pro-inflammatory cytokines was an important pathological cause for sudden death or meningitis induced by $S$. suis infection (20). Besides, the IFN- $\gamma$ response was also confirmed to be responsible for causing high mortality of STSLS (21). These experiments suggested that inhibition of the exaggerated inflammatory response could improve the outcome of STSLS. However, these findings seemed conflict with a previous study that pre-administration of IL- $1 \beta$ increased neutrophil and monocyte numbers and bactericidal activity, and then facilitate to control S. suis challenge (22). Therefore, inflammatory response may play complicate roles during S. suis infection.

Since its discovery in 2000, triggering receptor expressed on myeloid cells-1 (TREM-1) has been described as a critical immunomodulator in several inflammatory disorders (23). Infection with S. suis also induced TREM-1 expression (24), indicating the signaling involved in this infectious disease. Here, we present the current progresses of the immunoregulatory functions of TREM- 1 on acute infectious diseases and highlight the essential roles of TREM-1 on the development of STSLS.

\section{FUNCTION OF TREM-1 ON THE DEVELOPMENT OF INFECTIOUS DISEASES}

Triggering receptor expressed on myeloid cells-1 was firstly identified on lipopolysaccharide (LPS)-stimulated neutrophils and monocytes (25), and then confirmed to be highly expressed on granulocytes, DCs, and natural killer cells and lowly expressed on T and B cells (26). TREM-1, belonging to the Ig superfamily, is a cell surface-activating receptor with a single extracellular V-type Ig-like domain, a transmembrane region containing charged lysine residues and a short cytoplasmic tail lacking signaling motifs $(27,28)$. TREM- 1 can amplify toll-like receptor (TLR)-initiated responses against microbial challenges, enhancing the inflammatory response through interaction with an adaptor protein, DNAX-activating protein of $12 \mathrm{kDa}$ (DAP12) $(23,25,29)$. Due to its key role on enhancement of the inflammatory response, TREM-1 was recognized as an important regulator of innate immunity in sepsis (23, 30-33), septic shock (34-36), autoimmune arthritis (37), chronic inflammatory disorders $(38)$, inflammatory bowel disease $(39,40)$, and corneal inflammation (41).

Despite these previous findings, the results regarding the requirement of TREM-1 for controlling of microbial infections are controversial. TREM-1 contributed to neutrophilic infiltration, induction of pro-inflammatory cytokines, and the disease severity, but it could not obviously affect pathogen clearance during Leishmania major, influenza virus or Legionella pneumophila infection (42). By contrast, TREM-1 played an important role in controlling dissemination of Kelbsiella pneumoniae and improvement of survival in a model of a Klebsiella pneumoniae liver abscess (43). Another example for the contribution of TREM-1 to killing pathogen was the infectious model on Streptococcus pneumoniae with trem $1 / 3^{-/-}$mice (44) or agonistic TREM-1 antibody (45). TREM-1 was confirmed to play a role on secretion of cytokines and chemokines, neutrophils influx, clearance of Streptococcus pneumoniae, and improved survival $(44,45)$. Moreover, TREM-1/3 deficiency also increased local and systemic cytokine production, decreased the transepithelial migration of neutrophils into the airspace, and increased mortality during Pseudomonas aeruginosa infection (46). Therefore, these studies suggest that the roles of TREM-1-mediated immune responses to infection are very complicate.

\section{SOLUBLE FORM OF TREM-1 (STREM-1) AND INFECTIOUS DISEASE}

Apart from the membrane-bound form of TREM-1, a $27-\mathrm{kDa}$ glycosylated peptide, corresponding to the sTREM-1, has been found in body fluids of infected individuals $(30,47)$. Two hypotheses have been proposed to explain the origin of sTREM1: alternative splicing of TREM-1 mRNA (48) and proteolytic cleavage(s) of mature, membrane-anchored TREM-1 (49). With a general matrix metalloproteinase inhibitor, Gomez-Pina et al. demonstrated that metalloproteinases were responsible for shedding of the TREM-1 ectodomain through proteolytic cleavage of its long juxtamembrane linker (50).

The clinical significance of sTREM-1 has been confirmed in several studies in which sTREM-1 was detected in patients with chronic obstructive pulmonary disease (51), peptic ulcer disease (52), severe sepsis (53), septic shock (30), or inflammatory bowel disease (54). Now, sTREM-1 is recognized as a diagnostic and prognostic biomarker in patients with septic shock (55), neonatal sepsis (56), and Streptococcus pyogenes-induced sepsis (32).

At present, the function of sTREM is not fully understood. It is possible that sTREM-1 may negatively regulate receptor signaling through neutralization of the ligands, which is supported by the findings that the TREM-1 signaling could be significantly inhibited by a fusion protein containing the TREM-1 extracellular domain and human IgG1 Fc fragment (23) or the recombinant TREM-1 extracellular domain (57).

\section{SIGNALING FOR TREM-1 EXPRESSION}

Triggering receptor expressed on myeloid cells- 1 could be induced in response to various ligands, such as LPS $(25,58)$, bacteria $(23,41)$, and viruses $(59,60)$. In LPS-stimulated RWA264.7 cells, the transcription of TREM-1 was found positively and negatively regulated by NF- $\kappa$ B and PU.1 (61). In macrophages, LPS-induced TREM-1 expression was mediated, at least partly, by endogenous prostaglandins E2 followed by EP4 and cAMP, protein kinase A, p38 MAPK, and PI3K-mediated signaling (62). The expression of TREM-1 could also be inhibited by prostaglandins D2 and cyclopentanone prostaglandins PGJ2 and 15-dPGJ2, which was 
through activation of $\mathrm{Nrf2}$ and inhibition of NF- $\mathrm{KB}$. These provided a novel mechanism by which these prostaglandins show anti-inflammatory effects (63).

Based on the analysis of the trem 1 promoter, Hosoda et al. demonstrated that the cAMP response element (CRE) and NF- $\kappa B$-binding site in the mouse TREM-1 promoter regulated the basal TREM-1 transcription positively and negatively, respectively (64). In addition, CRE and NF- $\kappa \mathrm{B}$ possibly participated in the LPS-induced upregulation of TREM-1 promoter activity. AP-1 also seemed to be involved in the LPS-induced TREM-1 transcription through the interaction with phosphorylated c-fos/c-Jun (64).

Interestingly, TREM-1 expression in response to lipoteichoic acid is MyD88 dependent, and the expression induced by LPS is mediated by the TRIF signaling but not by MyD88, which suggest that signaling for TREM-1 induction is dependent on the specific TLR ligands (65).

\section{TREM-1 LIGANDS AND SIGNALING}

Activation of TREM-1 signaling is initiated when binding of the ligand to the receptor, which triggers the association and phosphorylation of the immunoreceptor tyrosine-based activation motif of the adaptor protein DAP12, resulting in the recruitment and activation of the non-receptor tyrosine kinase Syk. Syk, in turn, activates the downstream signaling molecules including PI3K, PLC $\gamma$, ERK1/2, and MAP kinases to induce the production of inflammatory chemokines and cytokines, such as IL-8 and myeloperoxidase (MPO), in neutrophils and IL-8, MCP-1, and TNF-alpha in monocytes $(29,66-68)$. In addition, TREM-1 also regulates macrophage survival through Bcl-2 (69), alters the dynamics of pulmonary IRAK-M expression, and improves host defense during pneumococcal pneumonia (45).

Identification of TREM-1 ligands is very important for understanding the nature of TREM-1 signaling. Gibot et al. first revealed that a TREM-1 ligand was induced on murine granulocytes during experimental peritonitis and sepsis (35). Interestingly, the surface glycoprotein of filoviruses was identified as a ligand for TREM-1 (70). Because endogenous signals released from necrotic cells could augment inflammatory responses through TREM-1, identification of the endogenous ligands would be more informative. HSP-70 and HMGB-1 from LPS-induced necrotic cell lysates might function as ligands for TREM-1, although the interaction between these proteins and TREM-1 was not confirmed in that study (71). Through screening hematopoietic cells for specific binding of a recombinant soluble fusion protein consisting of the extracellular domain of human TREM-1, Haselmayer et al. indicated that the natural ligand for TREM-1 was located on the surface of platelets (72). Considering the contribution of interaction between platelets and immune cells to the development of sepsis $(73,74)$, further identification of the ligands for TREM-1 activation in platelets was performed. Actin was identified as a TREM-1-interacting protein, and actin could activate inflammatory responses in a TREM-1-dependent manner (75). Since actin is a cellular cytoskeleton protein, there was a conflict about whether actin could be distributed on the cell surface. In fact, distribution of actin on the surface of platelets could be detected even in the resting state (76). Therefore, platelets did provide surface actin for TREM-1 recognition to activate signaling. In addition, HMGB1 was also confirmed as a TREM-1 ligand, which regulated Kupffer cell activation and development of hepatocellular carcinoma (77). The peptidoglycan recognition protein 1 (PGLYRP1) of neutrophils was also recognized as a functional ligand for TREM-1 (78). Until now, HMGB1, PGLYRP1, and extracellular actin have been identified as endogenous ligands for TREM-1 $(75,77,78)$, which indicated that various proteins could be served as activate signal for TREM-1 signaling. Interestingly, all the identified endogenous ligands for TREM-1 were involved in the inflammatory conditions. PGLYRP1 could form homodimers for its antimicrobial activity and could be induced in response to the infection (79); HMGB1 and actin are the cellular proteins and could be released from the cells in inflammatory conditions $(76,80)$. The characteristics of these ligands for TREM-1 provided an image of how TREM-1 signaling can be activated to control infection or cause severe disease (Figure 1).

In the resting state (Figure 1A), two reasons to confirm that TREM-1 signaling could not be activated by the surface actin on platelets: one reason is that TREM-1 expression is not induced in normal conditions; the other reason is that activation of TREM-1 on neutrophils by the surface actin on platelet requires the interaction of both cells, which is selectin/integrin dependent (72).

By contrast, low-level stimulation could activate neutrophils or monocytes and then induce expression of TREM-1 through various pattern-recognition receptors (Figure 1B). Then, the active immune cells could interact with platelets through selectin/ integrin dependent, which would further provide the condition for TREM-1 activation: the surface actin on platelets. In addition, the secreted PGLYRP1 and HMGB1 from host cells would also provide the endogenous signals for TREM-1 activation. This inflammatory condition mediated by TREM-1 signaling is required for some pathogen clearance $(43,44,81)$.

However, if the pathogen could not be controlled by the inflammatory cells, the overwhelming stimulation might be presented. Then, TREM-1 expression would be induced significantly. Furthermore, the stimulation could further cause actin and HMGB1 to be released from the dying host cells, which would provide a large quantity of ligands for TREM-1 activation to cause progressive systemic inflammatory responses, resulting in severe inflammation.

\section{A PROTECTIVE ROLE OF TREM-1 ON S. suis INFECTION}

Through transcriptional analysis on the swine response to S. suis infection, Li et al. found that the expression of TREM-1 was induced and that a few inflammatory genes were also highly expressed (24). Using a recombinant TREM-1 extracellular domain or an agonistic TREM-1 antibody as an inhibitor or activator of signaling, Yang et al. found that blocking TREM-1 signaling could not improve the survival of mice experiencing S. suis-induced septic shock (81). This finding is inconsistent with the effects of blocking TREM-1 signaling on sepsis or septic shock caused by other pathogens $(30-33,35,36)$. By contrast, they also found that the activation of TREM-1 signaling significantly 


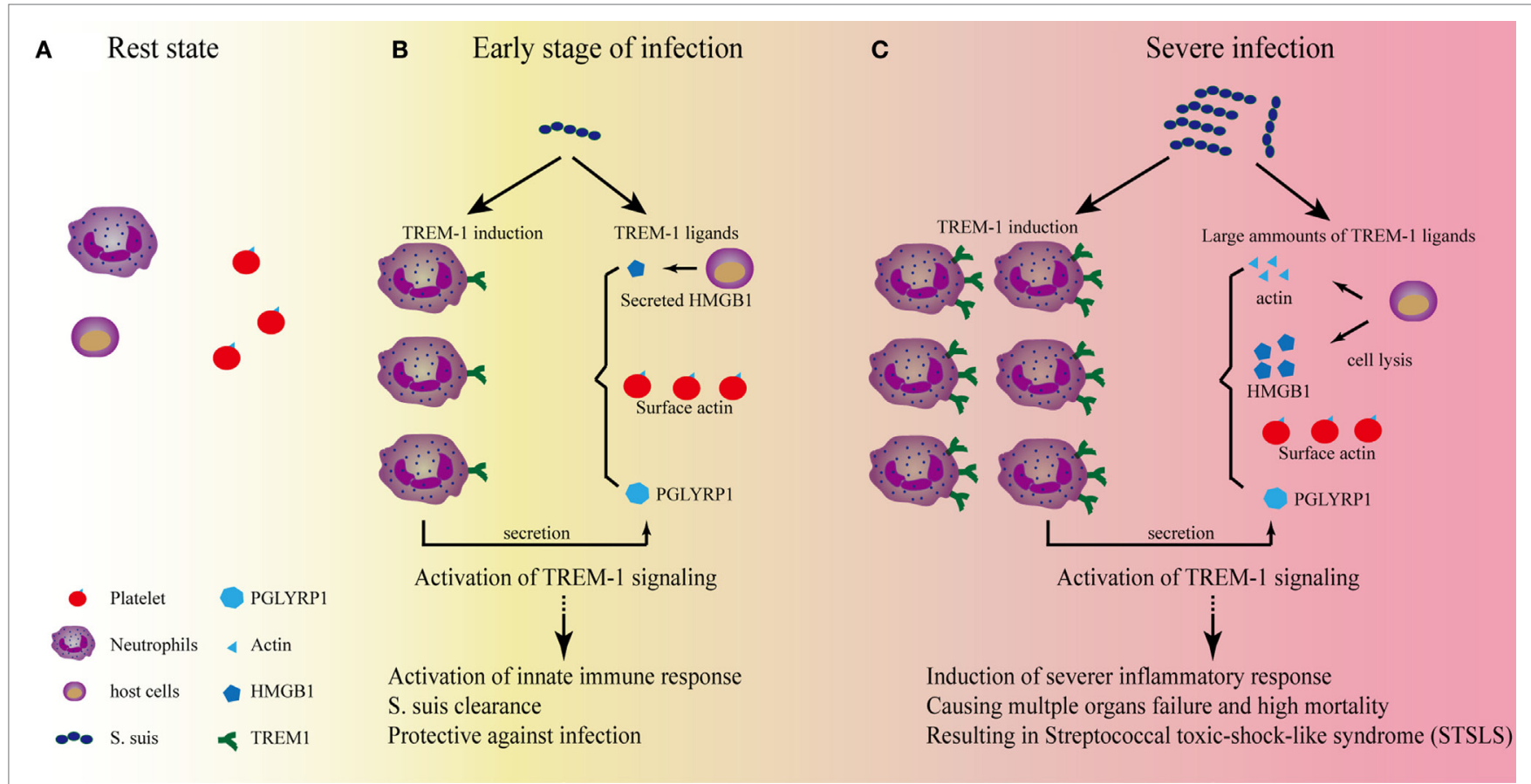

FIGURE 1 | The role of triggering receptor expressed on myeloid cells-1 (TREM-1) signaling on the development of streptococcal toxic-shock-like syndrome (STSLS) caused by Streptococcus suis. (A) In the resting state, two reasons to confirm that TREM-1 signaling could not be activated by the surface actin on platelets: One reason is that TREM-1 expression is not induced; the other reason is that activation of TREM-1 on neutrophils by the surface actin on platelet requires the interaction of both cells, which is selectin/integrin dependent. Therefore, the signaling does not occur. (B) At the early stage of $S$. suis infection, TREM-1 expression is induced through various pattern-recognition receptors, such as toll-like receptor (TLR)2, TLR4, TLR6, and so on. The activated host cells could also secrete HMGB1 or peptidoglycan recognition protein 1 (PGLYRP1), which could serve as ligands for TREM-1 activation. In addition, the activated neutrophils could interact with platelets which could further provide surface actin for TREM-1 activation. The activation of TREM-1 signaling is essential for further activation of neutrophils and monocytes, which are important for bacterial clearance. At this stage, if $S$. suis could be significantly killed by these innate immune cells, the infection would be under control. (C) Severe infection would occur if the bacterial could resist the clearance. The Chinese epidemic $S$. suis strain has developed many strategies to resist the early killings, and the quick propagation of $S$. suis would provide more ligands for TLR activation to induce a significantly high level of TREM-1 expression. In addition, necrosis of host cells due to the infection of $S$. suis would provide much more ligands (such as actin and HMGB1) to activate TREM-1 signaling to cause severe inflammation. Ultimately, a TREM-1-mediated severe inflammatory response results in the cytokine storm, multiple organs failure, and high mortality - the characteristics of STSLS.

improved the survival of mice infected with S. suis (81). These results indicated a protective role of TREM-1 on S. suis infection.

Furthermore, Yang et al. also noticed that TREM-1 blockage could intensify rather than inhibit the severe inflammatory response to $S$. suis infection, while activation could reduce inflammatory response (81). These results are confusing and give a contradictory function for TREM-1 signaling (23). However, the analysis on bacteria clearance indicated that the pro-inflammatory cytokine levels correlated well with the bacteria quantity in vivo, which suggested that blocking TREM-1 signaling may affect $S$. suis clearance, resulting in exacerbate inflammation (81).

Neutrophils played a very important role in controlling $S$. suis infection $(82,83)$, and an analysis indicated that TREM-1 signaling could significantly improve MPO level and neutrophils quantity in the blood during S. suis infection (81). Thus, the analysis further provided an explanation of how TREM-1 signaling provided a protective role of TREM-1 on S. suis infection: TREM-1 activation enhanced the activation of neutrophils and then contributed to the clearance of pathogen. Thus, TREM-1 blockage would inhibit inflammatory response and the activation of neutrophils, which would further reduce the clearance of
S. suis. These would increase bacteria quantity and further cause severe inflammation to ultimately result in adverse outcomes of S. suis infection.

\section{CONTRIBUTION OF TREM-1 TO STSLS}

Triggering receptor expressed on myeloid cells-1 plays an essential role on S. suis clearance (81), and TREM-1 blockage alone cannot rescue the host from the infection. To directly evaluate the role of TREM-1 on causing severe inflammation, an inhibitor of TREM-1 signaling was used in the presence of antibiotics, although the treatment effectiveness on $S$. suis infection remains controversial (18). Treatment with ampicillin alone could kill bacterial efficiently and also reduce the inflammatory cytokine response; however, it cannot significantly improve survival rates (57). These findings are similar to the outcomes of the clinical treatment of pigs and humans during S. suis infection. However, killing the bacteria and blocking the TREM-1-mediated inflammatory response at the same time could effectively alleviate the severe inflammation and protect the host against epidemic S. suis infection (57). Thus, these results indicate that TREM-1 signaling 
also contributes to the development of severe inflammation and STSLS. Undoubtedly, TREM-1 blockage in the presence of effective antibiotics would be a valuable treatment for STSLS.

\section{CONCLUSION}

Streptococcus suis infection may induce the expression of TREM-1 through various receptor, such as TLR2 (84-86), TLR4 (87), TLR6 (88), and so on, although the pattern-recognition receptor mainly responsible for STSLS remains to be identified $(85,89)$. At the early stage of infection (Figure 1B), TREM-1 recognizes the natural ligands (such as surface actin on the platelets) and activate neutrophils, which is essential for bacterial clearance (81). If the bacteria can be significantly killed by neutrophils, the infection will be under control. However, the Chinese epidemic strain has evolved many strategies to evade killing by host immune cells, such as resistance of phagocytosis (83) and acidic stress in lysosomes and endosomes (90), evading entrapment and killing by neutrophil extracellular traps $(91,92)$, resistance of complementmediated killing $(93,94)$, and so on. If S. suis successfully resisted killing, the quick propagation of bacteria will provide much more ligands for the activation of pattern-recognition receptors

\section{REFERENCES}

1. Segura M. Streptococcus suis: an emerging human threat. J Infect Dis (2009) 199:4-6. doi:10.1086/594371

2. Wertheim HF, Nghia HD, Taylor W, Schultsz C. Streptococcus suis: an emerging human pathogen. Clin Infect Dis (2009) 48:617-25. doi:10.1086/596763

3. Gottschalk M, Xu J, Lecours M-P, Grenier D, Fittipaldi N, Segura M. Streptococcus suis infections in humans: what is the prognosis for Western countries? (Part I). Clin Microbiol Newsl (2010) 32:89-96. doi:10.1016/j. clinmicnews.2010.06.001

4. Gottschalk M. Streptococcocis. 10th ed. In: Zimmerman J, Karriker L, Ramirez A, Schwartz K, Stevenson G, editors. Diseases of Swine. Ames, IA: Blackwell Publishing (2012). p. 841-55.

5. Goyette-Desjardins G, Auger JP, Xu J, Segura M, Gottschalk M. Streptococcus suis, an important pig pathogen and emerging zoonotic agent-an update on the worldwide distribution based on serotyping and sequence typing. Emerg Microbes Infect (2014) 3:e45. doi:10.1038/emi.2014.45

6. Huong VT, Ha N, Huy NT, Horby P, Nghia HD, Thiem VD, et al. Epidemiology, clinical manifestations, and outcomes of Streptococcus suis infection in humans. Emerg Infect Dis (2014) 20:1105-14. doi:10.3201/eid2007.131594

7. Suankratay C, Intalapaporn P, Nunthapisud P, Arunyingmongkol K, Wilde H. Streptococcus suis meningitis in Thailand. Southeast Asian J Trop Med Public Health (2004) 35:868-76.

8. Mai NT, Hoa NT, Nga TV, Linh Le D, Chau TT, Sinh DX, et al. Streptococcus suis meningitis in adults in Vietnam. Clin Infect Dis (2008) 46:659-67. doi:10.1086/527385

9. Huang YT, Teng LJ, Ho SW, Hsueh PR. Streptococcus suis infection. J Microbiol Immunol Infect (2005) 38:306-13.

10. Smith TC, Capuano AW, Boese B, Myers KP, Gray GC. Exposure to Streptococcus suis among US swine workers. Emerg Infect Dis (2008) 14:1925-7. doi:10.3201/eid1412.080162

11. Fowler HN, Brown P, Rovira A, Shade B, Klammer K, Smith K, et al. Streptococcus suis meningitis in swine worker, Minnesota, USA. Emerg Infect Dis (2013) 19:330-1. doi:10.3201/eid1902.120918

12. Tang J, Wang C, Feng Y, Yang W, Song H, Chen Z, et al. Streptococcal toxic shock syndrome caused by Streptococcus suis serotype 2. PLoS Med (2006) 3:e151. doi:10.1371/journal.pmed.0030151

13. Yu H, Jing H, Chen Z, Zheng H, Zhu X, Wang H, et al. Human Streptococcus suis outbreak, Sichuan, China. Emerg Infect Dis (2006) 12:914-20. doi:10.3201/ eid1206.051194 to induce high levels of TREM-1 expression. In addition, necrosis of host cells due to the infection will also provide more ligands (such as actin and HMGB1) to activate TREM-1 signaling to cause severe inflammation. Ultimately, a TREM-1-mediated severe inflammatory response results in the cytokine storm, multiple organs failure, and high mortality-characteristics of STSLS (Figure 1C). Therefore, TREM-1 signaling plays protective and pathogenic roles on STSLS.

\section{AUTHOR CONTRIBUTIONS}

All authors listed have made a substantial, direct, and intellectual contribution to the work and approved it for publication.

\section{FUNDING}

This work was supported by the National Key Research and Development Program of China (2017YFD0500204), the National Natural Science Foundation of China (31272544), the Natural Science Foundation of Hubei Province (2015CFA041, 2016CFA015), and the fundamental research funds for the central university (2662015PY221).

14. Manzin A, Palmieri C, Serra C, Saddi B, Princivalli MS, Loi G, et al. Streptococcus suis meningitis without history of animal contact, Italy. Emerg Infect Dis (2008) 14:1946-8. doi:10.3201/eid1412.080679

15. Callejo R, Prieto M, Salamone F, Auger JP, Goyette-Desjardins G, Gottschalk M. A typical Streptococcus suis in man, Argentina, 2013. Emerg Infect Dis (2014) 20:500-2. doi:10.3201/eid2003.131148

16. Choi SM, Cho BH, Choi KH, Nam TS, Kim JT, Park MS, et al. Meningitis caused by Streptococcus suis: case report and review of the literature. J Clin Neurol (2012) 8:79-82. doi:10.3988/jcn.2012.8.1.79

17. Zalas-Wiecek P, Michalska A, Grabczewska E, Olczak A, Pawlowska M, Gospodarek E. Human meningitis caused by Streptococcus suis. J Med Microbiol (2013) 62:483-5. doi:10.1099/jmm.0.046599-0

18. Lun ZR, Wang QP, Chen XG, Li AX, Zhu XQ. Streptococcus suis: an emerging zoonotic pathogen. Lancet Infect Dis (2007) 7:201-9. doi:10.1016/ S1473-3099(07)70001-4

19. Ye C, Zheng H, Zhang J, Jing H, Wang L, Xiong Y, et al. Clinical, experimental, and genomic differences between intermediately pathogenic, highly pathogenic, and epidemic Streptococcus suis. J Infect Dis (2009) 199:97-107. doi:10.1086/594370

20. Lachance C, Segura M, Dominguez-Punaro MC, Wojewodka G, De Sanctis JB, Radzioch D, et al. Deregulated balance of omega- 6 and omega- 3 polyunsaturated fatty acids following infection by the zoonotic pathogen Streptococcus suis. Infect Immun (2014) 82:1778-85. doi:10.1128/IAI.01524-13

21. Lachance C, Gottschalk M, Gerber PP, Lemire P, Xu J, Segura M. Exacerbated type II interferon response drives hypervirulence and toxic shock by an emergent epidemic strain of Streptococcus suis. Infect Immun (2013) 81:1928-39. doi:10.1128/IAI.01317-12

22. Shi J, Goodband RD, Chengappa MM, Nelssen JL, Tokach MD, Mcvey DS, et al. Influence of interleukin-1 on neutrophil function and resistance to Streptococcus suis in neonatal pigs. J Leukoc Biol (1994) 56:88-94. doi:10.1002/ jlb.56.1.88

23. Bouchon A, Facchetti F, Weigand MA, Colonna M. TREM-1 amplifies inflammation and is a crucial mediator of septic shock. Nature (2001) 410:1103-7. doi:10.1038/35074114

24. Li R, Zhang A, Chen B, Teng L, Wang Y, Chen H, et al. Response of swine spleen to Streptococcus suis infection revealed by transcription analysis. BMC Genomics (2010) 11:556. doi:10.1186/1471-2164-11-556

25. Bouchon A, Dietrich J, Colonna M. Cutting edge: inflammatory responses can be triggered by TREM-1, a novel receptor expressed on neutrophils and monocytes. J Immunol (2000) 164:4991-5. doi:10.4049/jimmunol.164.10.4991 
26. Matesanz-Isabel J, Sintes J, Llinas L, De Salort J, Lazaro A, Engel P. New B-cell CD molecules. Immunol Lett (2011) 134:104-12. doi:10.1016/j. imlet.2010.09.019

27. Radaev S, Kattah M, Rostro B, Colonna M, Sun PD. Crystal structure of the human myeloid cell activating receptor TREM-1. Structure (2003) 11:1527-35. doi:10.1016/j.str.2003.11.001

28. Kelker MS, Foss TR, Peti W, Teyton L, Kelly JW, Wuthrich K, et al. Crystal structure of human triggering receptor expressed on myeloid cells 1 (TREM-1) at 1.47 A. J Mol Biol (2004) 342:1237-48. doi:10.1016/j.jmb.2004.07.089

29. Dower K, Ellis DK, Saraf K, Jelinsky SA, Lin LL. Innate immune responses to TREM-1 activation: overlap, divergence, and positive and negative cross-talk with bacterial lipopolysaccharide. J Immunol (2008) 180:3520-34. doi:10.4049/jimmunol.180.5.3520

30. Gibot S, Kolopp-Sarda MN, Bene MC, Bollaert PE, Lozniewski A, Mory F, et al. A soluble form of the triggering receptor expressed on myeloid cells-1 modulates the inflammatory response in murine sepsis. J Exp Med (2004) 200:1419-26. doi:10.1084/jem.20040708

31. Wang F, Liu S, Wu S, Zhu Q, Ou G, Liu C, et al. Blocking TREM-1 signaling prolongs survival of mice with Pseudomonas aeruginosa induced sepsis. Cell Immunol (2012) 272:251-8. doi:10.1016/j.cellimm.2011.10.006

32. Horst SA, Linner A, Beineke A, Lehne S, Holtje C, Hecht A, et al. Prognostic value and therapeutic potential of TREM-1 in Streptococcus pyogenes-induced sepsis. J Innate Immun (2013) 5:581-90. doi:10.1159/000348283

33. van Bremen T, Dromann D, Luitjens K, Dodt C, Dalhoff K, Goldmann T, et al. Triggering receptor expressed on myeloid cells-1 (TREM-1) on blood neutrophils is associated with cytokine inducibility in human E. coli sepsis. Diagn Pathol (2013) 8:24. doi:10.1186/1746-1596-8-24

34. Nathan C, Ding A. TREM-1: a new regulator of innate immunity in sepsis syndrome. Nat Med (2001) 7:530-2. doi:10.1038/87846

35. Gibot S, Buonsanti C, Massin F, Romano M, Kolopp-Sarda MN, Benigni F, et al. Modulation of the triggering receptor expressed on the myeloid cell type 1 pathway in murine septic shock. Infect Immun (2006) 74:2823-30. doi:10.1128/IAI.74.5.2823-2830.2006

36. Gibot S, Massin F, Marcou M, Taylor V, Stidwill R, Wilson P, et al. TREM-1 promotes survival during septic shock in mice. Eur JImmunol (2007) 37:456-66. doi:10.1002/eji.200636387

37. Murakami Y, Akahoshi T, Aoki N, Toyomoto M, Miyasaka N, Kohsaka H. Intervention of an inflammation amplifier, triggering receptor expressed on myeloid cells 1, for treatment of autoimmune arthritis. Arthritis Rheum (2009) 60:1615-23. doi:10.1002/art.24554

38. Schenk M, Bouchon A, Seibold F, Mueller C. TREM-1 - expressing intestinal macrophages crucially amplify chronic inflammation in experimental colitis and inflammatory bowel diseases. J Clin Invest (2007) 117:3097-106. doi:10.1172/JCI30602

39. Park JJ, Cheon JH, Kim BY, Kim DH, Kim ES, Kim TI, et al. Correlation of serum-soluble triggering receptor expressed on myeloid cells-1 with clinical disease activity in inflammatory bowel disease. Dig Dis Sci (2009) 54:1525-31. doi:10.1007/s10620-008-0514-5

40. Genua M, Rutella S, Correale C, Danese S. The triggering receptor expressed on myeloid cells (TREM) in inflammatory bowel disease pathogenesis. J Transl Med (2014) 12:293. doi:10.1186/s12967-014-0293-Z

41. Wu M, Peng A, Sun M, Deng Q, Hazlett LD, Yuan J, et al. TREM-1 amplifies corneal inflammation after Pseudomonas aeruginosa infection by modulating toll-like receptor signaling and Th1/Th2-type immune responses. Infect Immun (2011) 79:2709-16. doi:10.1128/IAI.00144-11

42. Weber B, Schuster S, Zysset D, Rihs S, Dickgreber N, Schurch C, et al. TREM-1 deficiency can attenuate disease severity without affecting pathogen clearance. PLoS Pathog (2014) 10:e1003900. doi:10.1371/journal.ppat.1003900

43. Lin YT, Tseng KY, Yeh YC, Yang FC, Fung CP, Chen NJ. TREM-1 promotes survival during Klebsiella pneumoniae liver abscess in mice. Infect Immun (2014) 82:1335-42. doi:10.1128/IAI.01347-13

44. Hommes TJ, Hoogendijk AJ, Dessing MC, Van't Veer C, Florquin S, Colonna M, et al. Triggering receptor expressed on myeloid cells-1 (TREM-1) improves host defence in pneumococcal pneumonia. J Pathol (2014) 233:357-67. doi:10.1002/path.4361

45. Lagler H, Sharif O, Haslinger I, Matt U, Stich K, Furtner T, et al. TREM-1 activation alters the dynamics of pulmonary IRAK-M expression in vivo and improves host defense during pneumococcal pneumonia. J Immunol (2009) 183:2027-36. doi:10.4049/jimmunol.0803862
46. Klesney-Tait J, Keck K, Li X, Gilfillan S, Otero K, Baruah S, et al. Transepithelial migration of neutrophils into the lung requires TREM-1. J Clin Invest (2013) 123:138-49. doi:10.1172/JCI64181

47. Gibot S, Cravoisy A, Kolopp-Sarda MN, Bene MC, Faure G, Bollaert PE, et al. Time-course of sTREM (soluble triggering receptor expressed on myeloid cells)-1, procalcitonin, and C-reactive protein plasma concentrations during sepsis. Crit Care Med (2005) 33:792-6. doi:10.1097/01.CCM.0000159089. $16462.4 \mathrm{~A}$

48. Gingras MC, Lapillonne H, Margolin JF. TREM-1, MDL-1, and DAP12 expression is associated with a mature stage of myeloid development. $\mathrm{Mol}$ Immunol (2002) 38:817-24. doi:10.1016/S0161-5890(02)00004-4

49. Klesney-Tait J, Turnbull IR, Colonna M. The TREM receptor family and signal integration. Nat Immunol (2006) 7:1266-73. doi:10.1038/ni1411

50. Gomez-Pina V, Soares-Schanoski A, Rodriguez-Rojas A, Del Fresno C, Garcia F, Vallejo-Cremades MT, et al. Metalloproteinases shed TREM-1 ectodomain from lipopolysaccharide-stimulated human monocytes. J Immunol (2007) 179:4065-73. doi:10.4049/jimmunol.179.6.4065

51. Phua J, Koay ES, Zhang D, Tai LK, Boo XL, Lim KC, et al. Soluble triggering receptor expressed on myeloid cells-1 in acute respiratory infections. Eur Respir J (2006) 28:695-702. doi:10.1183/09031936.06.00005606

52. Koussoulas V, Vassiliou S, Demonakou M, Tassias G, GiamarellosBourboulis EJ, Mouktaroudi M, et al. Soluble triggering receptor expressed on myeloid cells (sTREM-1): a new mediator involved in the pathogenesis of peptic ulcer disease. Eur J Gastroenterol Hepatol (2006) 18:375-9. doi:10.1097/00042737-200604000-00010

53. Tao F, Peng L, Li J, Shao Y, Deng L, Yao H. Association of serum myeloid cells of soluble triggering receptor-1 level with myocardial dysfunction in patients with severe sepsis. Mediators Inflamm (2013) 2013:819246. doi:10.1155/ 2013/819246

54. Schenk M, Bouchon A, Birrer S, Colonna M, Mueller C. Macrophages expressing triggering receptor expressed on myeloid cells-1 are underrepresented in the human intestine. J Immunol (2005) 174:517-24. doi:10.4049/ jimmunol.174.1.517

55. Brenner T, Uhle F, Fleming T, Wieland M, Schmoch T, Schmitt F, et al. Soluble TREM-1 as a diagnostic and prognostic biomarker in patients with septic shock: an observational clinical study. Biomarkers (2017) 22:63-9. doi:10.1080/ 1354750X.2016.1204005

56. Adly AA, Ismail EA, Andrawes NG, El-Saadany MA. Circulating soluble triggering receptor expressed on myeloid cells-1 (sTREM-1) as diagnostic and prognostic marker in neonatal sepsis. Cytokine (2014) 65:184-91. doi:10.1016/j.cyto.2013.11.004

57. Yang C, Zhao J, Lin L, Pan S, Fu L, Han L, et al. Targeting TREM-1 signaling in the presence of antibiotics is effective against streptococcal toxic-shock-like syndrome (STSLS) caused by Streptococcus suis. Front Cell Infect Microbiol (2015) 5:79. doi:10.3389/fcimb.2015.00079

58. Haselmayer P, Daniel M, Tertilt C, Salih HR, Stassen M, Schild H, et al. Signaling pathways of the TREM-1- and TLR4-mediated neutrophil oxidative burst. J Innate Immun (2009) 1:582-91. doi:10.1159/000231973

59. Denner J, Eschricht M, Lauck M, Semaan M, Schlaermann P, Ryu H, et al. Modulation of cytokine release and gene expression by the immunosuppressive domain of gp41 of HIV-1. PLoS One (2013) 8:e55199. doi:10.1371/ journal.pone.0055199

60. Ruiz-Pacheco JA, Vivanco-Cid H, Izaguirre-Hernandez IY, Estrada-Garcia I, Arriaga-Pizano L, Chacon-Salinas R, et al. TREM-1 modulation during early stages of dengue virus infection. Immunol Lett (2014) 158:183-8. doi:10.1016/j. imlet.2014.01.003

61. Zeng H, Ornatowska M, Joo MS, Sadikot RT. TREM-1 expression in macrophages is regulated at transcriptional level by NF-kappaB and PU.1. Eur J Immunol (2007) 37:2300-8. doi:10.1002/eji.200737270

62. Murakami Y, Kohsaka H, Kitasato H, Akahoshi T. Lipopolysaccharideinduced up-regulation of triggering receptor expressed on myeloid cells-1 expression on macrophages is regulated by endogenous prostaglandin E2. J Immunol (2007) 178:1144-50. doi:10.4049/jimmunol.178.2.1144

63. Syed MA, Joo M, Abbas Z, Rodger D, Christman JW, Mehta D, et al. Expression of TREM-1 is inhibited by PGD2 and PGJ2 in macrophages. Exp Cell Res (2010) 316:3140-9. doi:10.1016/j.yexcr.2010.08.009

64. Hosoda H, Tamura H, Kida S, Nagaoka I. Transcriptional regulation of mouse TREM-1 gene in RAW264.7 macrophage-like cells. Life Sci (2011) 89:115-22. doi:10.1016/j.lfs.2011.05.007 
65. Zheng $\mathrm{H}$, Heiderscheidt CA, Joo M, Gao X, Knezevic N, Mehta D, et al. MYD88-dependent and -independent activation of TREM-1 via specific TLR ligands. Eur J Immunol (2010) 40:162-71. doi:10.1002/eji.200839156

66. Fortin CF, Lesur O, Fulop T Jr. Effects of TREM-1 activation in human neutrophils: activation of signaling pathways, recruitment into lipid rafts and association with TLR4. Int Immunol (2007) 19:41-50. doi:10.1093/intimm/dxl119

67. Tessarz AS, Cerwenka A. The TREM-1/DAP12 pathway. Immunol Lett (2008) 116:111-6. doi:10.1016/j.imlet.2007.11.021

68. Roe K, Gibot S, Verma S. Triggering receptor expressed on myeloid cells-1 (TREM-1): a new player in antiviral immunity? Front Microbiol (2014) 5:627. doi:10.3389/fmicb.2014.00627

69. Yuan Z, Syed MA, Panchal D, Joo M, Colonna M, Brantly M, et al. Triggering receptor expressed on myeloid cells 1 (TREM-1)-mediated Bcl-2 induction prolongs macrophage survival. J Biol Chem (2014) 289:15118-29. doi:10.1074/ jbc.M113.536490

70. Mohamadzadeh M, Coberley SS, Olinger GG, Kalina WV, Ruthel G, Fuller CL, et al. Activation of triggering receptor expressed on myeloid cells-1 on human neutrophils by Marburg and Ebola viruses. J Virol (2006) 80:7235-44. doi:10.1128/JVI.00543-06

71. El Mezayen R, El Gazzar M, Seeds MC, Mccall CE, Dreskin SC, Nicolls MR. Endogenous signals released from necrotic cells augment inflammatory responses to bacterial endotoxin. Immunol Lett (2007) 111:36-44. doi:10.1016/ j.imlet.2007.04.011

72. Haselmayer P, Grosse-Hovest L, Von Landenberg P, Schild H, Radsak MP. TREM-1 ligand expression on platelets enhances neutrophil activation. Blood (2007) 110:1029-35. doi:10.1182/blood-2007-01-069195

73. Vincent JL, Yagushi A, Pradier O. Platelet function in sepsis. Crit Care Med (2002) 30:S313-7. doi:10.1097/00003246-200205001-00022

74. Semple JW, Italiano JE Jr, Freedman J. Platelets and the immune continuum. Nat Rev Immunol (2011) 11:264-74. doi:10.1038/nri2956

75. Fu L, Han L, Xie CY, Li WK, Lin L, Pan S, et al. Identification of extracellular actin as a ligand for triggering receptor expressed on myeloid cells-1 signaling. Front Immunol (2017) 8:917. doi:10.3389/fimmu.2017.00917

76. Bachvaroff RJ, Miller F, Rapaport FT. Appearance of cytoskeletal components on the surface of leukemia cells and of lymphocytes transformed by mitogens and Epstein-Barr virus. Proc Natl Acad Sci U S A (1980) 77:4979-83. doi:10.1073/pnas.77.8.4979

77. Wu J, Li J, Salcedo R, Mivechi NF, Trinchieri G, Horuzsko A. The proinflammatory myeloid cell receptor TREM-1 controls Kupffer cell activation and development of hepatocellular carcinoma. Cancer Res (2012) 72:3977-86. doi:10.1158/0008-5472.CAN-12-0938

78. Read CB, Kuijper JL, Hjorth SA, Heipel MD, Tang X, Fleetwood AJ, et al. Cutting edge: identification of neutrophil PGLYRP1 as a ligand for TREM-1. J Immunol (2015) 194:1417-21. doi:10.4049/jimmunol.1402303

79. Osanai A, Sashinami H, Asano K, Li SJ, Hu DL, Nakane A. Mouse peptidoglycan recognition protein PGLYRP-1 plays a role in the host innate immune response against Listeria monocytogenes infection. Infect Immun (2011) 79:858-66. doi:10.1128/IAI.00466-10

80. Wang H, Bloom O, Zhang M, Vishnubhakat JM, Ombrellino M, Che J, et al. HMG-1 as a late mediator of endotoxin lethality in mice. Science (1999) 285:248-51. doi:10.1126/science.285.5425.248

81. Yang C, Chen B, Zhao J, Lin L, Han L, Pan S, et al. TREM-1 signaling promotes host defense during the early stage of infection with highly pathogenic Streptococcus suis. Infect Immun (2015) 83:3293-301. doi:10.1128/IAI. 00440-15

82. Busque P, Higgins R, Senechal S, Marchand R, Quessy S. Simultaneous flow cytometric measurement of Streptococcus suis phagocytosis by polymorphonuclear and mononuclear blood leukocytes. Vet Microbiol (1998) 63:229-38. doi:10.1016/ S0378-1135(98)00216-8

83. Chabot-Roy G, Willson P, Segura M, Lacouture S, Gottschalk M. Phagocytosis and killing of Streptococcus suis by porcine neutrophils. Microb Pathog (2006) 41:21-32. doi:10.1016/j.micpath.2006.04.001

84. Graveline R, Segura M, Radzioch D, Gottschalk M. TLR2-dependent recognition of Streptococcus suis is modulated by the presence of capsular polysaccharide which modifies macrophage responsiveness. Int Immunol (2007) 19:375-89. doi:10.1093/intimm/dxm003

85. Zheng H, Dominguez-Punaro MC, Segura M, Lachance C, Rivest S, Xu J, et al. Toll-like receptor 2 is partially involved in the activation of murine astrocytes by Streptococcus suis, an important zoonotic agent of meningitis. J Neuroimmunol (2011) 234:71-83. doi:10.1016/j.jneuroim.2011.02.005

86. Zhang Q, Yang Y, Yan S, Liu J, Xu Z, Yu J, et al. A novel pro-inflammatory protein of Streptococcus suis 2 induces the toll-like receptor 2-dependent expression of pro-inflammatory cytokines in RAW 264.7 macrophages via activation of ERK1/2 pathway. Front Microbiol (2015) 6:178. doi:10.3389/ fmicb.2015.00178

87. Bi L, Pian Y, Chen S, Ren Z, Liu P, Lv Q, et al. Toll-like receptor 4 confers inflammatory response to suilysin. Front Microbiol (2015) 6:644. doi:10.3389/ fmicb.2015.00644

88. Zheng H, Luo X, Segura M, Sun H, Ye C, Gottschalk M, et al. The role of tolllike receptors in the pathogenesis of Streptococcus suis. Vet Microbiol (2012) 156:147-56. doi:10.1016/j.vetmic.2011.10.014

89. Lachance C, Segura M, Gerber PP, Xu J, Gottschalk M. Toll-like receptor 2-independent host innate immune response against an epidemic strain of Streptococcus suis that causes a toxic shock-like syndrome in humans. PLoS One (2013) 8:e65031. doi:10.1371/journal.pone.0065031

90. Gruening P, Fulde M, Valentin-Weigand P, Goethe R. Structure, regulation, and putative function of the arginine deiminase system of Streptococcus suis. J Bacteriol (2006) 188:361-9. doi:10.1128/JB.188.2.361-369.2006

91. de Buhr N, Neumann A, Jerjomiceva N, Von Kockritz-Blickwede M, Baums CG. Streptococcus suis DNase SsnA contributes to degradation of neutrophil extracellular traps (NETs) and evasion of NET-mediated antimicrobial activity. Microbiology (2014) 160:385-95. doi:10.1099/mic.0.072199-0

92. Zhao J, Pan S, Lin L, Fu L, Yang C, Xu Z, et al. Streptococcus suis serotype 2 strains can induce the formation of neutrophil extracellular traps (NETs) and evade trapping. FEMS Microbiol Lett (2015) 362:fnv022. doi:10.1093/femsle/ fnv022

93. Lecours MP, Gottschalk M, Houde M, Lemire P, Fittipaldi N, Segura M. Critical role for Streptococcus suis cell wall modifications and suilysin in resistance to complement-dependent killing by dendritic cells. J Infect Dis (2011) 204:919-29. doi:10.1093/infdis/jir415

94. Roy D, Grenier D, Segura M, Mathieu-Denoncourt A, Gottschalk M. Recruitment of factor H to the Streptococcus suis cell surface is multifactorial. Pathogens (2016) 5:E47. doi:10.3390/pathogens5030047

Conflict of Interest Statement: The authors declare that the research was conducted in the absence of any commercial or financial relationships that could be construed as a potential conflict of interest.

Copyright (C) 2018 Han, Fu, Peng and Zhang. This is an open-access article distributed under the terms of the Creative Commons Attribution License (CC BY). The use, distribution or reproduction in other forums is permitted, provided the original author(s) and the copyright owner are credited and that the original publication in this journal is cited, in accordance with accepted academic practice. No use, distribution or reproduction is permitted which does not comply with these terms. 